\section{Los recursos humanos de salud en Chile: el desafío pendiente de la reforma}

\section{Claudio A. Méndez ${ }^{1}$}

Forma de citar: Méndez CA. Los recursos humanos de salud en Chile: el desafío pendiente de la reforma. Rev Panam Salud Publica. 2009;26(3):276-80.

Palabras clave: gestión en salud; personal de salud; política de salud; recursos humanos; reforma de la atención de salud; Chile; América Latina.

Instituto de Salud Pública, Facultad de Medicina, Universidad Austral de Chile. La correspondencia se debe dirigir a Instituto de Salud Pública, Facultad de Medicina, Universidad Austral de Chile. Avenida Senador Acharán Arce s/n, Campus Isla Teja, CP 567, Valdivia, Chile. Correo electrónico claudiomendez@uach.cl
Algunos autores han destacado la dificultad de posicionar a los recursos humanos en salud como un aspecto substancial de los procesos de reforma de la salud (1). Tal afirmación se da precisamente en un escenario de crisis global del capital humano, producto de décadas de fallas en la formación de profesionales sanitarios, condiciones laborales y salarios deficientes, con la consecuente emigración de recursos humanos, y en general una mala gestión de este componente crítico para el buen funcionamiento de los sistemas de salud. En este contexto, al que hay que sumarle la insuficiencia de conocimientos acerca de los procesos de planificación y desarrollo de políticas y programas, no se puede desconocer la urgencia de que los sistemas de salud trabajen conjuntamente para dar prioridad al problema de los recursos humanos en los procesos de reforma actuales y futuros (2).

Dentro de estos esfuerzos conjuntos, es preciso reconocer que la importancia del recurso humano no solo radica en la función fundamental de proveer servicios de salud, sino que además representa cerca de $60 \%$ del gasto en los sistemas (3), convirtiéndolo en un componente clave en el momento de planificar, organizar y ejecutar políticas públicas en salud (4). Los procesos de reforma, por lo tanto, deben incluir políticas de recursos humanos que destaquen su financiamiento y sus funciones en los nuevos modelos asistenciales, así como normas claras para los mecanismos de contratación y regulación (5).

En América Latina y el Caribe, los procesos de reforma del sector salud - provocados principalmente por los cambios epidemiológicos, demográficos y sociopolíticos de las últimas décadas- dieron un fuerte impulso a la provisión de prestaciones de salud mediante el aseguramiento de la accesibilidad, la calidad de la atención, la descentralización, la separación de funciones y el creciente papel rector de los ministerios de salud $(6,7)$. No obstante, la ausencia de estudios de costobeneficio políticos, económicos y sociales no permitió frenar el ensanchamiento de la brecha de inequidad en los servicios, ni lograr una verdadera descentralización en las estrategias de implantación de las políticas sanitarias (8). Así, aun con el indudable empuje que dieron estos procesos a ciertos aspectos de los sistemas y servicios de salud del continente, en el caso del capital humano la nueva perspectiva economicista propició una fuerte resistencia a la introducción de modelos de atención orientados al mercado; una crítica a la inadecuada adopción de los procesos de reforma y la ausencia de capacitación a la fuerza de trabajo, la falta de información respecto a los cambios, y una legislación reguladora débil que no aseguraba calidad profesional (9).

Con todo, las reformas de salud representaron la oportunidad de movilizarse en la búsqueda de nuevas 
y mejores condiciones de trabajo y beneficios salariales (10), incluso cuando estos procesos posteriormente se orientarían principalmente a la productividad, la evaluación del desempeño, los sistemas de incentivos y nuevas formas de contratación (11), postergando así, entre otros, la desigual distribución del recurso humano en salud, la necesidad de reducir la concentración de especialidades y prestaciones de alta complejidad, y el aumento en la migración de profesionales (12).

\section{El capital humano de salud en Chile}

En 2004 el Ministerio de Salud (MINSAL) de Chile contaba con 25542 médicos, de los cuales 2276 provenían formados de otros países, en particular Cuba, Colombia y Eduador. La tasa de médicos por beneficiarios del Fondo Nacional de Salud (FONASA) era de 8,45/10 000, con 2,3 médicos generales y 4,9 médicos especialistas por 10000 beneficiarios. En cuanto a su distribución nacional, los médicos graduados en las especialidades básicas de cirugía general, medicina interna, obstetricia y ginecología, pediatría y psiquiatría, se concentraban mayoritariamente en las regiones Metropolitana, de Valparaíso y del Bío-Bío, las más pobladas del país (13).

No obstante esta situación, entre 1990 y 2006 se registró un explosivo aumento de - en orden decreciente de expansión- centros formadores de kinesiólogos, fonoaudiólogos, terapeutas ocupacionales, enfermeras, nutricionistas, odontólogos y médicos, en consonancia con un incremento paralelo de nuevos centros de educación superior en todo el país (14). Sin embargo, en el caso de la profesión médica, para algunos autores el desbalance entre médicos generales y especialistas sigue constituyendo uno de los principales desafíos del país (15).

En relación al funcionamiento del sector salud chileno, los problemas de gestión se reflejaban en una débil integración institucional, y ausencia de coordinación y complementariedad entre los niveles de atención y los recursos humanos, desaprovechados en razón de un bajo nivel de rendimiento, con alto ausentismo, poca flexibilidad y bajas remuneraciones (16). Los cambios introducidos a partir de la modernización de la gestión pública en la década de los noventa favorecieron al capital humano en salud a partir del reconocimiento del desempeño y la capacitación como elementos críticos de la carrera funcionaria. Sin embargo, la antigüedad siguió siendo un eje importante para lograr la permanencia de los funcionarios en las instituciones del sector. Durante el mismo período, en el nivel de políticas, el MINSAL elaboró un marco de orientación para desarrollar los recursos humanos, con énfasis en la transformación de la cultura organizacional de los servicios (17).

Actualmente el país transita un proceso de reforma del sector sanitario, concebida a partir de los principios del derecho a la salud, la equidad, la solidaridad en el financiamiento, la eficiencia en el uso de los recursos y la participación social. En el caso del personal, la reforma se propone desarrollar y fortalecer el capital humano a través de la redefinición de las profesiones médicas, la formación y actualización continuas, la acreditación periódica y el estudio de mecanismos e incentivos para la carrera funcionaria (18). No obstante estas consideraciones en el diseño, los desafíos identificados por la Organización Panamericana de la Salud en cuanto al recurso humano en salud se encuentran plenamente vigentes en la actual fase de implementación del proceso reformista (12).

El presente artículo analiza el desafío de la política de recursos humanos en Chile en el actual escenario de implementación de la reforma de salud. La primera parte aborda el proceso de reforma de salud del país, para posteriormente desarrollar sus principales aspectos de la política del personal de salud durante la fase de implementación y los desafíos de una tarea que todavía está pendiente.

\section{La reforma de salud en Chile}

Los cambios introducidos al sistema sanitario nacional en las décadas de los setenta y los ochenta provocaron un severo desfinanciamiento del sector, con especial impacto en los establecimientos hospitalarios, que registraron un deterioro significativo en la calidad de sus servicios, y en la financiación de los recursos humanos (19). Por tal motivo, una vez restablecido el régimen democrático, los primeros gobiernos de la Concertación de Partidos por la Democracia, a través del MINSAL, dirigieron sus esfuerzos a recuperar la inversión en salud y mejorar aspectos de infraestructura y accesibilidad a los servicios (20). En 2002, la reforma orientó sus objetivos hacia la definición de garantías explícitas en salud y exigibles para los ciudadanos, el mejoramiento de los modelos de atención y gestión del sistema, así como el establecimiento de solidaridad en el financiamiento, mediante el aporte de la población con menor riesgo de enfermarse en favor de la que posee un riesgo mayor. ${ }^{2}$

La primera acción en este sentido fue la promulgación en 2003 de la Ley 19 988, que "Establece financiamiento necesario para asegurar los objetivos sociales prioritarios del gobierno" (21), cuya aprobación aseguraba la financiación de la reforma de salud. Posteriormente se promulgaron los siguientes cuerpos legales: la Ley 19.937 de Autoridad Sanitaria y Gestión que define la nueva estructura del MINSAL (22), la Ley 19966 que establece el Régimen General de Garantías en Salud (23), la Ley 20.015 que modifica la ley de Institución de Salud Previsional (ISAPRE) $\mathrm{N}^{\circ}$ 18.433 (24) y el proyecto de ley de deberes y derechos de los usuarios, que todavía se encuentra en trámite legislativo en el Congreso de la Nación (cuadro 1).

Actualmente los problemas de salud priorizados bajo el régimen de garantías explícitas en salud, establecido por la reforma, ascienden a 56 , con una meta para el año 2010 de 80 patologías priorizadas.

\footnotetext{
La iniciativa de financiamiento solidario no prosperó en las negociaciones legislativas, por lo cual finalmente fue excluida del proceso.
} 
CUADRO 1. Cuerpos de ley y contenidos que integran la reforma de la salud en Chile

\begin{tabular}{|c|c|}
\hline Ley & Contenido \\
\hline \multirow{3}{*}{$\begin{array}{l}\text { Ley } 19.937 \text { de Autoridad Sanitaria } \\
\text { y Gestión }\end{array}$} & Crea la Subsecretaría de Redes Asistenciales, la cual coordina toda la red asistencial del país. \\
\hline & $\begin{array}{l}\text { Crea la Subsecretaría de Salud Pública, encargada de las normas sanitarias y la vigilancia } \\
\text { epidemiológica. }\end{array}$ \\
\hline & $\begin{array}{l}\text { Crea los Establecimientos Autogestionados en Red, los cuales serán centros con sistema de medición } \\
\text { de costos, calidad de servicios prestados y satisfacción de los usuarios. }\end{array}$ \\
\hline \multirow[t]{3}{*}{$\begin{array}{l}\text { Ley } 19.966 \text { que establece el Régimen } \\
\text { de Garantías en Salud }\end{array}$} & $\begin{array}{l}\text { Establece garantías explícitas referentes a acceso, calidad, protección financiera y oportunidad } \\
\text { con que deben ser otorgadas las prestaciones asociadas a un conjunto prioritario de programas, } \\
\text { enfermedades y condiciones de salud. }\end{array}$ \\
\hline & $\begin{array}{l}\text { Establece la elaboración de estudios para determinar prioridades en salud y de intervenciones } \\
\text { que consideren la situación de salud de la población y su costo-efectividad. }\end{array}$ \\
\hline & $\begin{array}{l}\text { Contempla la realización de estudios epidemiológicos, de carga de enfermedad, económicos } \\
\text { y de demanda potencial. }\end{array}$ \\
\hline $\begin{array}{l}\text { Ley } 20.015 \text { que modifica la Ley } \\
\text { de ISAPRE }\end{array}$ & Establece cambios en relación al funcionamiento de las ISAPRES. \\
\hline \multirow[t]{2}{*}{$\begin{array}{l}\text { Proyecto de ley de derechos } \\
\text { y deberes de los usuarios }\end{array}$} & $\begin{array}{l}\text { Aborda aspectos del derecho del paciente al trato digno, a la información y a la autonomía } \\
\text { del usuario, así como regulación a la investigación clínica, consultas y reclamos. }\end{array}$ \\
\hline & Involucra deberes de los usuarios enfocados a la responsabilidad ciudadana. \\
\hline
\end{tabular}

a En trámite legislativo en el Congreso Nacional.

\section{Política de recursos humanos en el programa de reforma}

Si bien el programa de reforma del sector salud de Chile inserta algunos cambios dirigidos al desempeño de los recursos humanos, hay componentes clave relacionados con los trabajadores de la salud que no fueron considerados.

Los cambios introducidos. Los componentes de la reforma de salud que se relacionan con los recursos humanos - presentes en la Ley de Autoridad Sanitaria y Gestión- se dirigen a la introducción de incentivos al desempeño colectivo y a la asignación de responsabilidades gerenciales. [La Ley no contempla, sin embargo, planes y políticas de recursos humanos enmarcados en el proceso de transformación que experimenta el sector.]

Mediante la inclusión de los componentes mencionados, lo que se busca es fortalecer la gestión de los recursos humanos e instrumentar medidas que incentiven a los profesionales más idóneos a permanecer en el sector público (10). No obstante, tales acciones son extemporáneas al diseño de la reforma, principalmente por la separación de las dimensiones de la cultura organizacional y las competencias para la gestión dentro del proceso de reforma. Sin desconocer los alcances de los lineamientos reformistas referentes a la política de recursos humanos, la carrera dentro de las organizaciones todavía sigue ligada a los años de servicio y no a los méritos profesionales en el campo de la gerencia y la gestión. Además, los cambios impulsados por la reforma para consolidar la descentralización y los mecanismos de mercado en la gestión de las instituciones sanitarias (25) han sido más precavidos en cuanto a la política de recursos humanos, fundamentalmente por la fuerte influencia de las asociaciones gremiales, tanto médicas como no profesionales, que no fueron involucradas en el proceso de reforma (26).

Las ausencias en la puesta en marcha. La reforma del sector salud en general carece de consideraciones sustanciales en cuanto al personal sanitario, nueva infraestructura, equipos y consumibles, lo cual se traduce en una de las grandes limitaciones al momento de ejecutar cualquier proceso de cambio (27). Tampoco aborda la introducción de nuevas formas de gestión para los directivos de acuerdo al nuevo escenario, ni una planificación del desarrollo profesional en consenso con los actores gremiales y políticos del sector (28). No incluye asimismo un sistema para determinar la necesidad de profesionales y técnicos en base a indicadores epidemiológicos y sanitarios, ni los requerimientos que conlleva la puesta en práctica tanto de las garantías en salud como los cambios introducidos en la gestión y la autoridad sanitaria (29). Lamentablemente, tampoco promueve una mejor combinación de destrezas en la formación de equipos para la gestión de las instituciones de salud (30).

Es sabido que las estrategias para planificar la demanda, la movilidad profesional y la identificación de los factores que diferencian y afectan la productividad de los distintos tipos de trabajadores (31) son prioritarias en cualquier reforma dirigida a priorizar las intervenciones en salud y la autonomía de ciertos tipos de establecimientos sanitarios. Estos componentes, sin embargo, también fueron soslayados en privilegio de la adopción de las garantías en salud.

Considerando lo expuesto, la casi imperceptible presencia del recurso humano en las políticas públicas de salud en favor de temas de financiamiento y cam- 
bios estructurales, se podría traducir en la oposición a las nuevas ideas de la reforma por parte de grupos de profesionales, la ausencia de nuevas capacidades administrativas y de gestión, y la reticencia general de los trabajadores de la salud a los cambios (32). Si la transformación de los sistemas de salud ha de ser plenamente exitosa, será imprescindible entonces reformular políticas de recursos humanos que se articulen con las nuevas estrategias del sector como un componente esencial para superar los inevitables desafíos que se irán presentando durante el tránsito de la reforma.

La apuesta en la fase de puesta en marcha. Ante la ausencia de componentes descritos como primordiales para alinear a los recursos humanos en la instrumentación de la reforma sanitaria de Chile, se pueden identificar tres escenarios posibles durante la fase de implementación que podrían facilitar una mejor armonización del capital humano con los cambios y modelos de gestión propuestos.

El primero consiste en mantener la actual y difusa política de recursos humanos, en donde los lineamientos estén desagregados entre las orientaciones para una nueva cultura organizacional, por un lado, y las responsabilidades y competencias para la gestión de los servicios de salud por el otro, evitando de esta forma una reestructuración en plena reforma que podría derivar en un complejo escenario de negociación con los gremios del sector. El segundo escenario sería dejar en manos de los servicios de salud del país la misión de readecuar las necesidades de sus cuadros, en razón de los requerimientos territoriales de la reforma. Vale señalar, no obstante, que tal escenario se dificulta por la excesiva centralización de las políticas públicas en salud del país. Finalmente, un tercer escenario - a más a largo plazo - consistiría en convocar a todas las partes interesadas en los recursos humanos en salud, con el fin de trazar lineamientos consensuados para establecer una política que ayude a conducir e implementar los cambios generados por la reforma.

\section{Comentarios finales}

La reforma del sector de la salud en Chile ha introducido transformaciones no exentas de complejidades técnicas y operativas. En tal sentido, la ausencia de una política de recursos humanos que acompañe $-\mathrm{y}$, sin dudas, facilite - la fase de implantación de todos los componentes de la reforma constituye una lección aún no aprendida sobre un aspecto fundamental para el éxito de procesos de cambio tan complejos como son las reformas de salud. La transformación de los sistemas de salud, si ha de arrojar los resultados deseados, debe llevarse a cabo mediante la planificación y el establecimiento de mecanismos que hagan hincapié en la productividad del capital humano, así como en la identificación de especialidades que se consideren necesarias para optimizar los servicios. No hay dudas de que un enfoque tal contribuiría a que la reforma cuente con una adherencia mucho mayor por parte del personal de la salud. Dado que la fase de ejecución de la reforma sanitaria de Chile aún se encuentra en los primeros pasos, todavía se está a tiempo de fortalecer su contenido integrándola más cabalmente con la dimensión de los recursos humanos. El desafío de conseguirlo sin dudas va a estar en manos de los expertos responsables de diseñar las estrategias y las políticas públicas en salud.

\section{SYNOPSIS}

\section{Human resources for health in Chile: the reform's pending challenge}

Omission of human resources from health policy development has been identified as a barrier in the health sector reform's adoption phase. Since 2002, Chile's health care system has been undergoing a transformation based on the principles of health as a human right, equity, solidarity, efficiency, and social participation. While the reform has set forth the redefinition of the medical professions, continuing education, scheduled accreditation, and the introduction of career development incentives, it has not considered management options tailored to the new setting, a human resources strategy that has the consensus of key players and sector policy, or a process for understanding the needs of health care staff and professionals. However, there is still time to undo the shortcomings, in large part because the reform's implementation phase only recently has begun. Overcoming this challenge is in the hands of the experts charged with designing public health strategies and policies.

Key words: health management; health personnel; health policy; human resources; health care reform; Chile; Latin America.

\section{REFERENCIAS}

1. Buchan J. What difference does ("good") HRM make? Human Resources for Health 2004;2:6. Hallado en: http:// www.human-resources-health.com/ content/2/1/6. Acceso el 11 agosto de 2009.

2. Chen L, Evans T, Anand S, Baufford JT, Brown $\mathrm{H}$, Chowdhury M, et al. Human resources for health: overcoming the crisis. Lancet. 2004;364:1984-90.
3. Arroyo J. Los sistemas descentrados de recursos humanos en salud: el caso del Perú, 1990-2005. Ciência \& Saúde Coletiva. 2006;11(4):1063-72.

4. Muñoz F, Lopez-Acuña D, Halverson $P$, Guerra de Macedo C, Hanna W, Larrieu $\mathrm{M}$, et al. Las funciones esenciales de la salud pública: un tema emergente en las reformas del sector de la salud. Rev Panam Salud Publica. 2000; 8(1-2):126-34.
5. Pierantoni C. As reformas do Estado, da saúde e recursos humanos: limites e possibilidades. Ciência e Saúde Coletiva. 2001;6(2):341-60.

6. Homedes N, Ugalde A. Why neoliberal health reform have failed in Latin America. Health Policy. 2005;71:83-96.

7. Infante A, de la Mata I, López-Acuña D. Reforma de los sistemas de salud en América Latina y el Caribe: situación y 
tendencias. Rev Panam Salud Publica. 2000;8(1-2):13-20.

8. Ugalde A, Homedes N. Las reformas neoliberales del sector salud: déficit gerencial y alienación del recurso humano en América Latina. Rev Panam Salud Publica. 2005;17(3):210-20.

9. Homedes N, Ugalde A. Human resources: the Cinderella of health sector reform in Latin America. Human Resources for Health 2005, 3:1. Hallado en: http:/ / www.human-resources-health. com/content $/ 3 / 1 / 1$. Acceso el 30 diciembre de 2008.

10. Rigoli F, Dussault G. The interface between health sector reform and human resources in health. Human Resources for Health 2003; 1:9. Hallado en: http: www.human-resources-health.com/ content/1/1/9. Acceso el 30 diciembre de 2008.

11. Brito PE. Impacto de las reformas del sector de la salud sobre los recursos humanos y la gestión laboral. Rev Panam Salud Publica. 2000;8(1-2):43-54

12. Organización Panamericana de la Salud. Desarrollo y Fortalecimiento de la Gestión de los Recursos Humanos en el Sector Salud. $43^{\circ}$ Consejo Directivo, Washington DC E. U. A; 24-28 de septiembre de 2001.

13. Román $\mathrm{O}$, Acuña M, Señoret M. Disponibilidad de médicos en Chile al año 2004. Rev Méd Chile. 2006;134:1057-64.

14. González D. Formación de recursos humanos profesionales en salud. Rev Chil Salud Pública. 2006;10(1):60-3.

15. Román O, Pineda S, Señoret M. Perfil y número de médicos generales que requiere el país. Rev Méd Chile. 2007;135: 1209-15.

16. Sojo A. Reformas de gestión en la salud pública en Chile. Santiago de Chile, Comisión Económica para América Latina y el Caribe, 1996. Series Políticas Sociales No. 13.
17. Ministerio de Salud de Chile. Políticas de desarrollo de las personas en la Red Asistencial del Sector Salud. Santiago: Ministerio de Salud; 2005.

18. Sandoval H. Mejor Salud para los Chilenos: fundamentos sanitarios, políticos y financieros de la necesidad y oportunidad de hacer una reforma al Sistema de Salud Chileno. Cuad Med Soc. 2004 43(1):5-20.

19. Jimenez J, Bossert T. Chile's health sector reform: lessons from four reform periods. Health Policy. 1995;32:155-66.

20. Artaza O, Montt J, Vásquez C. Estructura y recursos humanos en un hospital público chileno: una experiencia de cambio. Rev Panam Salud Publica. 1997; 2(5):342-51.

21. Ministerio de Hacienda de Chile. Ley No. 19.988 establece financiamiento necesario para asegurar los objetivos sociales prioritarios del gobierno. Hallado en: http:// www.bcn.cl/leyes/pdf/actualizado/ 213493.pdf. Acceso el 2 febrero de 2009.

22. Ministerio de Salud de Chile. Ley No. 19.937 de Autoridad Sanitaria y Gestión. Hallado en: http://www.bcn.cl/leyes/ pdf/actualizado/221629.pdf. Acceso el 6 enero de 2009.

23. Ministerio de Salud de Chile. Ley No. 19.966 Régimen General de Garantías en Salud. Hallado en: http:/ /www.bcn.cl/ leyes/pdf/actualizado/229834.pdf. Acceso el 6 enero de 2009.

24. Ministerio de Salud de Chile. Ley No. 20.015 Modifica la Ley 18.433 de la Ley de Instituciones de Salud Previsional (ISAPRE). Hallado en: http://www. bcn.cl/leyes/pdf/actualizado/238102. pdf. Acceso el 6 enero de 2009.

25. Lethbridge J. Public sector reform and demand for human resources for health. Human Resources for Health 2004, 2:15. Hallado en: http: www.human-resourceshealth.com/content/2/1/15. Acceso el 30 diciembre de 2008.
26. Lenz R. Proceso político de la reforma AUGE de salud en Chile: algunas lecciones para América Latina. Una mirada desde la economía política. Santiago de Chile, Corporación de Estudios para Latinoamérica, 2007. Serie estudios socio/ económicos No. 38

27. Sepúlveda-Alvarez C. El significado histórico de la reforma de la atención de salud en Chile. Cuad Méd Soc. 2004;43 (1):21-36

28. Martínez J, Martineau T. Rethinking human resources: an agenda for the millennium. Health Policy Plan. 1998;13(4): 345-58.

29. Dreesch N, Dolea C, Dal Poz M, Goubarev A, Adams O, Aregawi M, et al. An approach to estimating human resource requirements to achieve the Millennium Development Goals. Health Policy Plan. 2005;20:267-76.

30. Buchan J. Health sector reform and human resources: lessons from the United Kingdom. Health Policy Plan. 2000;15(3): 319-25.

31. Narasimhan $V$, Brown $H$, Pablos-Mendez A, Adams O, Dassault G, et al. Responding to the global human resources crisis. Lancet. 2004;363:1469-72.

32. Dussault G, Dubois C. Human resources for health policies: a critical component in health policies. Human Resources for Health 2003; 1:1. Hallado en: http:// www.human-resources-health.com/ content/1/1/1. Acceso el 30 diciembre de 2008 .

Manuscrito recibido el 2 de septiembre de 2008 Aceptado para publicación, tras revisión, el 5 de febrero de 2009. 